Review

\title{
Demographic Differences in the Quantity, Mass, and Anatomical Location of Ingested Plastic in Northern Fulmars (Fulmarus glacialis): A Review and Reconsideration of NE Pacific Ocean Samples
}

Gary W. Shugart ${ }^{1,}{ }^{*}$, Tessa G. Nania ${ }^{1,2}$

1. Slater Museum of Natural History, University of Puget Sound, Tacoma, Washington, 98416, US; E-Mail: gshugart@pugetsound.edu

2. Department of Biological Sciences, University of Puget Sound, Tacoma, Washington, 98416, US; E-Mail: tessa.georgina@gmail.com

* Correspondence: Gary W. Shugart; E-Mail: gshugart@pugetsound.edu

Academic Editor: Zed Rengel

Adv Environ Eng Res

2021, volume 2, issue 3

doi:10.21926/aeer.2103023
Received: July 09, 2021

Accepted: September 21, 2021

Published: September 29, 2021

\begin{abstract}
On the Washington and Oregon coast of the NE Pacific Ocean from 2008-2015, we found that juveniles represented $83 \%$ of Northern Fulmars that were beached. In comparison to older birds, juveniles averaged more mass and pieces of plastic in stomachs. This reflected relatively larger plastic loads in the cranial stomach section, the proventriculus, which we found was associated with relatively large accumulations in the caudal stomach section, the ventriculus. We estimate that the proventriculus could retain almost 10 times as much plastic as the ventriculus and that retention of proventricular plastic largely accounted for the difference in juveniles versus older birds. Our findings contrast with published Atlantic Ocean reports where the proportion of immatures was lower and plastic was retained mostly in ventriculi. The differences in demography and gastric distribution between NE Pacific and Atlantic fulmars may reflect the different sizes of plastic particles that were available. The preponderance of juveniles in NE Pacific Ocean samples and associated relatively large plastic loads overestimate the load for the species. Without accounting for age and gastric distribution, comparison to other regions is ill-advised. An unrecognized complication in
\end{abstract}

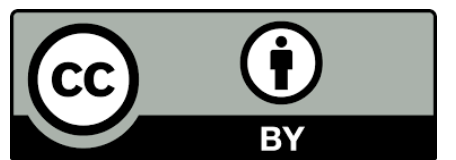

(C) 2021 by the author. This is an open access article distributed under the conditions of the Creative Commons by Attribution License, which permits unrestricted use, distribution, and reproduction in any medium or format, provided the original work is correctly cited. 
stomach-based sampling is that differences in size of plastic at spatiotemporal scales could affect the utility of stomach samples as bioindicators of oceanic plastic pollution.

\section{Keywords}

Fulmarus glacialis; fulmar; stomach plastic

\section{Introduction}

Plastic found in stomachs of dead seabirds has been used as a bioindicator of spatiotemporal trends in plastic pollution [1-10]. Although finding stomach plastic is now the norm in some seabird species (e.g., Northern Fulmar, Fulmarus glacialis), surprisingly little is known about dynamics of ingestion, retention, and elimination [3, 11-14]. In the absence of information on dynamics, assumptions are made regarding reasons for ingestion (mistakes, olfactory attraction, facilitation of digestion), retention (times relatively short or long, size dependent), and elimination (defecation, regurgitation) (see $[12,13,15]$ for reviews). Uncertainties regarding dynamics were expressed when stomach plastic was first documented in the 1980s [16-20].

Two factors that impinge on the above assumptions and data are the distribution of plastic in sections of the stomach and demographic profiles of samples [1, 10, 13, 21]. In retrospect, the first is significant because legacy protocols for presenting monitoring results pooled loads from the two sections of the stomach: the proventriculus and ventriculus. Pooling was done for expediency [1, 2 , 4] although the stomach sections differ in morphology, function, and physiology [13, 21, 22]. The proventriculus is thin-walled and expandable with up to 10 times the capacity of the thick-walled ventriculus (see below). Food first enters the proventriculus for storage or chemical digestion before passing into the ventriculus for grinding objects before passage into the intestine. One general finding throughout plastic studies is that if plastic is found, it is primarily in ventriculi $[13,17,21,23$, 24]. The significance of this is that the small size and thick walls of the ventriculus set an upper limit to the amount of plastic that could accumulate while the sphincter between the two stomach sections limits the size of particles that could enter the ventriculus [13, 22]. Thus, while providing a standard sampling unit, ventricular samples are limited in the number and size of particles while the expansive proventriculus could hold more and larger pieces [13, 21].

Noting that plastic must first pass through the proventriculus to enter the ventriculus, the lower incidence of plastic in proventriculi can only mean that it is cleared more frequently through regurgitation, passage into ventriculi, or both [13, 22]. Analyzing plastic from stomach sections separately could provide potentially useful information about temporal differences in the time plastic remains in residence in sections of the stomach $[7,13,25]$. The proventricular plastic load provides a relatively transient or ephemeral sample of recent ingestion while the ventricular load likely represents residual plastic that is retained for a longer period. In addition, the difference in capacity and relative short-term occurrence of the proventricular load relative to ventricular loads could impart considerable variation in pooled samples.

Noting the constraints on ventricular loads and that adults contain less plastic than immatures $[1,9,13,15,26,27]$, we surmised that the difference in the amount of proventricular plastic in pooled samples accounts for much of the variation in loads and the difference between age groups. 
In this paper, we confirm that some juveniles from the NE Pacific Coasts contain more proventricular plastic and associated greater plastic loads than in older birds. We then reconsider our NE Pacific samples in comparison to Atlantic fulmar samples relative to recent research on the availability of the sizes of available plastic.

\section{Materials and Methods}

\subsection{Study Area and Data}

Data from Terepocki et al. [13] examined the dynamics of plastic in two stomach sections while in this paper we examined data from a demographic perspective focusing on differences between juveniles and non-juveniles. The latter consisted of adults and immatures in pre-breeding cohorts (see Results). Plastic was extracted and processed using standard protocol $[13,28]$ and archived at the Slater Museum of Natural History, University of Puget Sound. An additional sample was added to these data from the 2014-2015 time period from fulmars obtained from the same Pacific coast beaches in Oregon and Washington (see [13]). A total of 197 stomach samples were analyzed and 195 were aged (Table 1).

Table 1 Distribution of Northern Fulmars by time period, age, and number without plastic (=No) and with plastic (=Yes) in six time periods in the Northeast Pacific Ocean coasts of Oregon and Washington.

\begin{tabular}{|c|c|c|c|c|c|c|c|c|c|c|}
\hline \multirow[b]{2}{*}{ Time-period } & \multicolumn{3}{|c|}{ Juvenile } & \multicolumn{3}{|c|}{ Non-Juvenile } & \multicolumn{3}{|c|}{ Undetermined } & \multirow[b]{2}{*}{ Grand Tota } \\
\hline & No & Yes & Total & No & Yes & Total & No & Yes & Total & \\
\hline 2008-2009 & & 6 & 6 & 1 & 5 & 6 & & & & 12 \\
\hline $2009-2010$ & 4 & 8 & 12 & 1 & 9 & 10 & & & & 22 \\
\hline $2010-2011$ & 4 & 65 & 69 & 1 & 3 & 4 & 2 & & 2 & 75 \\
\hline 2011-2012 & & 1 & 1 & 2 & 12 & 14 & & & & 15 \\
\hline $2012-2013$ & 1 & 16 & 17 & 1 & 1 & 2 & & & & 19 \\
\hline 2014-2015 & 11 & 40 & 51 & 1 & 2 & 3 & & & & 54 \\
\hline Total & 20 & 136 & 156 & 7 & 32 & 39 & 2 & & 2 & 197 \\
\hline$\%$ with plastic & & & 87.2 & & & 82.05 & & & & 85.2 \\
\hline
\end{tabular}

In addition to the stomach contents, we also inspected the intestines of the 2014-2015 sample. The intestine was removed, straightened, and bisected longitudinally, and flatten with the lumen exposed in a trough. The trough was placed on a slight incline the contents were washed into a 0.5 $\mathrm{mm}$ sieve. The contents were examined under a dissecting scope and processed using standard protocol $[13,28]$.

Along the NE Pacific Coast, juvenile fulmars are easily identified using criteria of no remex or rectrix molt, uniform unworn body plumage, diminutive testes or ovaries with no apparent ova (undifferentiated), and relatively large glandular-walled bursa of Fabricius ([29]; Figure 12 in [28]). Juveniles were hatched in July [30], and they would be 3-6 months post-fledgling when encountered. Fulmars follow a Simple Basic Molt Strategy [30], and lack of molt, except for some on head and neck, were obvious clues to the juvenile category that we verified with internal data. Immatures had a mix of old and new body feathers, remex or rectrix molt, and thin-walled non-glandular bursas. 
The bursa transitions from a glandular thick-walled structure to a membranous thin-walled structure probably during the second year of life, but could persist for a longer period. Adults had similar molt status but lacked a bursa. Gonads of non-breeding adults, which regress in size during the non-breeding period (see [31]) are probably similar to older immatures. Without bursa information, the criteria for designating immatures versus adults would be indefinite (see [1]), and older immatures could be categorized as adults or the reverse. In comparison to other studies (see Discussion) age categorizations might differ dependent on the experience of personnel and age classes might not be comparable if personnel with differing experience made determinations.

Bursas were examined by snipping the ventral lip of the cloaca opening, examining the caudal side of the uroproctodeal fold for an opening, and then inserting a blunt measuring tool to determine depth if an opening was apparent. The bursa was then opened ventrally and the interior was examined and classified as thick glandular wall or thin membranous wall. Width was also measured (see [32]).

\subsection{Statistical Analysis}

Data were analyzed using Minitab 17.3.1 [33]. Nonparametric tests were used and medians with quartiles were included in summaries in addition to arithmetic mean \pm SE for comparison to other studies. The two-sample signed-rank test referred to as Wilcoxon-Mann-Whitney or Mann-Whitney [34] test in Minitab reports the statistic of Wilcoxon $W$ accounting for ties as appropriate. Probabilities were two-tailed. Summaries and statistics are presented based on all birds including those with no plastic. This is the norm in stomach-based plastic summaries, but underestimates the loads in affected birds (see [35, 36]). Data for plastic mass and pieces are presented as arithmetic means and median following the protocol of van Franeker and Meijboom [1].

\section{Results}

The distribution of birds per year (Table 1 ) and month (Table 2 ) indicates that recovery of age groups differed between time periods and that most were recovered from October through November. Of the three age groups, mass and number of pieces of plastic differed (Table 3 ) (Kruskall-Wallis $2 \mathrm{H}=13.21, p=0.001$ ). Pair-wise comparisons indicated that juveniles differed from immatures and adults, but adults and immature were not significantly different. Adults and immatures were pooled in subsequent analysis. Juveniles comprised 85\% (156/195) of aged specimens. Of the juveniles, $87 \%(136 / 156)$ had retained plastic which did not differ from $82 \%$ (32/39) of non-juveniles with plastic (Chi-square $=0.668, p=0.407)$. Juveniles had more pieces $\left(W_{156}\right.$, $39=4,178, p<0.001)$ and greater total mass $\left(W_{156,39}=4,306, p<0.001\right)$ of plastic than non-juveniles (Figure 1). Two juveniles from the 2014-2015 period were extreme outliers with 26,251.6 mg and 466 pieces and 20,231.4 mg and 278 pieces (Table 3). However, these did not distort the comparisons since Mann-Whitney-Wilcoxon ranked comparisons were used. 
Table 2 Age distribution, cross indexed by month of recovery, of beached Northern Fulmars from the Oregon and Washington coast. See Table 1 for time periods.

\begin{tabular}{|c|c|c|c|c|c|c|c|c|c|c|c|c|c|c|c|}
\hline Age & Jul & Aug & Sep & Oct & Nov & Dec & Jan & Feb & Mar & Apr & Oct-Nov & Oct-Jan & Feb-Mar & Feb-Apr & Total \\
\hline Juvenile & 1 & & 1 & 52 & 70 & 12 & 8 & 1 & & & & 3 & 1 & 7 & 156 \\
\hline Non-juvenile & 6 & 5 & & 6 & 5 & 1 & & 1 & 1 & 1 & 2 & 5 & & 6 & 39 \\
\hline Undetermined & & & & 1 & & & 1 & & & & & & & & 2 \\
\hline Total & 7 & 5 & 1 & 59 & 75 & 13 & 9 & 2 & 1 & 1 & 2 & 8 & 1 & 13 & 197 \\
\hline
\end{tabular}

Table 3 Age distribution of beached Northern Fulmars from Oregon and Washington coasts showing comparative data used for pooling in age classes of juveniles and non-juveniles. The latter included immatures, adults, and seven birds designated Immature-Adult (Imm-Adult) could be older immatures or adults (see Methods). Two of undetermined ages were excluded. For all the minimum was zero. Q1 and Q3 are lower and upper quartiles about the median. $\mathrm{N}$ = sample size or number of stomachs and values refer to summary values for the sample not individual plastic pieces.

\begin{tabular}{lllllllll}
\hline Variable & Age & $\mathrm{N}$ & Mean & $\mathrm{SE}$ & $\mathrm{Q} 1$ & Median & Q3 & Max \\
\hline Mass $(\mathrm{mg})$ & Juvenile & 156 & 990 & 229 & 79 & 268 & 765 & 26,252 \\
& Immature & 10 & 164.8 & 62.1 & 5.0 & 51 & 304 & 570 \\
& Adult & 22 & 224.0 & 94.4 & 8.8 & 46 & 179 & 1,651 \\
& Imm-Adult & 7 & 226 & 102 & 14 & 141 & 305 & 880 \\
& & & & & & & & \\
Pieces & Juvenile & 156 & 31.7 & 4.15 & 4.0 & 17.0 & 41.5 & 466 \\
& Immature & 10 & 11.55 & 5.1 & 1.0 & 5.0 & 15.0 & 53 \\
& Adult & 22 & 9.05 & 2.2 & 1.0 & 6.5 & 12.0 & 44 \\
& Imm-Adult & 7 & 11.75 & 4.3 & 0.75 & 11.0 & 16.5 & 37 \\
\hline
\end{tabular}



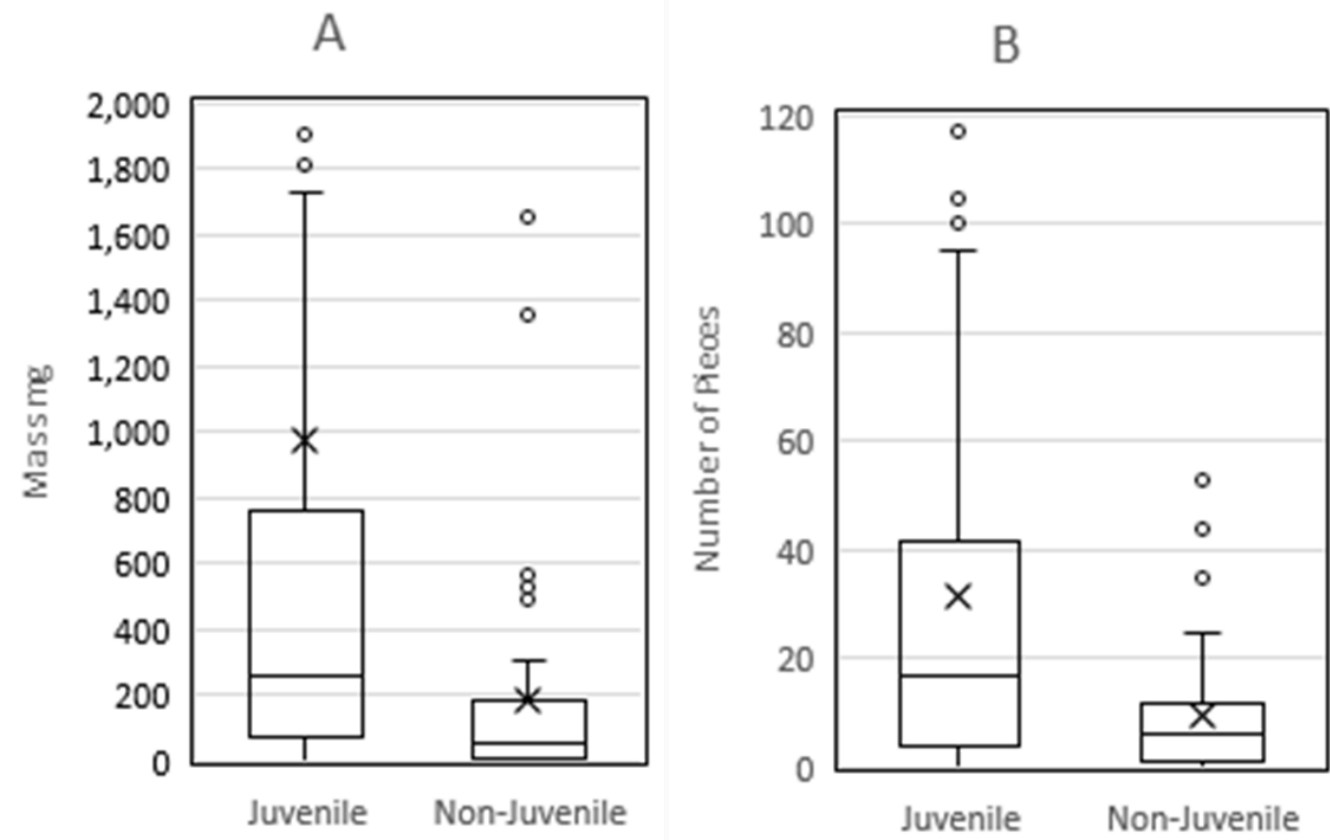

Figure 1 Plastic retained in Northern Fulmar proventriculi and ventriculi (pooled) from the NE Pacific Ocean coasts of Oregon in Washington for 2008-2015 by age. Differences in mass $(A)$ and pieces (B) between age groups were significant (see text). Shown are $1^{\text {st }}$ and $3^{\text {rd }}$ quartiles (box), median (line in box), mean ( $x$ ), outliers (circles), and vertical lines are 1-1/2 times the interquartile range. Extreme outliers were excluded from the figure to show the box more clearly.

The distribution of plastic in stomachs differed for ages with $51 \%(79 / 156)$ of juveniles retaining proventricular plastic compared to only $28 \%(11 / 39$ ) of non-juveniles (Chi-square $=6.319, p=0.012$. In contrast, there was no significant difference between the $87 \%(136 / 156)$ of juveniles and $82 \%$ (32/39) of non-juveniles that retained plastic in ventriculi (Chi-square $=1.225, p=0.268$ ). Only two fulmars, an adult (SDT43) and a juvenile (LUK20) had $15 \mathrm{mg} / 5$ pieces and $20 \mathrm{mg} / 1$ pieces, respectively, in the proventriculus with no plastic in the ventriculus.

Juveniles had greater mass and number of pieces in both proventriculi and ventriculi than nonjuveniles (Table 4, mass $W_{156,39}=16,552.50, p<0.001$, number of pieces $\left(W_{156,39}=16,424.00, p<\right.$ 0.001). These differences result in proventriculus/ventriculus ratios for juveniles of 1.8 for mass and 0.7 for pieces in contrast to non-juvenile ratios of 0.5 for mass and 0.1 for pieces (Table 4). Although large ventricular loads in association with the presence of proventricular plastic had been noted [13], the association with age was not recognized. This association of proventricular loads with larger ventricular loads with age was not apparent in non-juveniles (mass $W_{21,10}=318.00, p=0.460$; number of pieces $W_{21,10}=318.00, p=0.537$;) (Table 5 ). Similar amounts of plastic occurred in juveniles versus non-juveniles that only had ventricular plastic (mass $W_{57,21}=4,083.00, p=0.118$; number of pieces $W_{57,21}=4,034.00, p=0.252$ ) (Table 5). 
Table 4 Mass and pieces of plastic in 195 Northern Fulmars by age categories used in the study. All were from the NE Pacific coast and included empty stomachs by proventriculus (PV), ventriculus (V), and total plastic. Juv = Juvenile, Non Juv = Nonjuvenile; $\mathrm{Q} 1$ and $\mathrm{Q} 3$ are quartiles about the median.

\begin{tabular}{lllllllllll}
\hline $\begin{array}{l}\text { Mass } \\
\text { (mg) }\end{array}$ & Age & $\mathrm{N}$ & $\begin{array}{l}\text { \% with } \\
\text { plastic }\end{array}$ & Mean & SE & Min & Q1 & Median & Q3 & Max \\
\hline PV & Juv & 156 & 50.6 & 640 & 208 & 0 & 0 & 5 & 291 & 24,374 \\
& Non Juv & 39 & 28.2 & 60.1 & 39.4 & 0 & 0 & 0 & 5.1 & 1221 \\
V & Juv & 156 & 86.5 & 351.8 & 156 & 0 & 0 & 198.8 & 486.9 & $2,593.7$ \\
& Non Juv & 39 & 86.1 & 128.4 & 29.3 & 0 & 4.7 & 43.2 & 183.5 & 677 \\
Total & Juv & 156 & 87.2 & 990 & 229 & 0 & 29 & 268 & 765 & 26,252 \\
& Non Juv & 39 & 88.9 & 188.4 & 56.2 & 0 & 5 & 51 & 188 & 1,651 \\
\hline Pieces & & & & & & & & & & \\
\hline PV & Juv & 156 & 50.6 & 13.1 & 3.45 & 0 & 0 & 1 & 7 & 426 \\
& Non Juv & 39 & 28.2 & 0.97 & 0.4 & 0 & 0 & 0 & 1 & 13 \\
\multirow{2}{*}{ V } & Juv & 156 & 86.5 & 18.6 & 1.6 & 0 & 3.25 & 13 & 26.75 & 104 \\
& Non Juv & 39 & 86.1 & 8.5 & 1.8 & 0 & 1 & 4 & 12 & 48 \\
\multirow{2}{*}{ Total } & Juv & 156 & 87.2 & 31.7 & 4.15 & 0 & 4 & 17 & 41.5 & 466 \\
& Non Juv & 39 & 88.9 & 9.4 & 9.4 & 0 & 1 & 6 & 12 & 53 \\
\hline
\end{tabular}

Table 5 Juvenile and non-juvenile Northern Fulmars with the mass and pieces of plastic in their ventriculus comparing those with plastic versus those without plastic in their proventriculus (PV). Q1 and Q3 are quartiles about the median.

\begin{tabular}{lllllllll}
\hline Juveniles & N & Mean & SE & Min & Q1 & Median & Q3 & Max \\
\hline Without PV plastic, mg & 57 & 199.9 & 23.4 & 2.9 & 79.4 & 147.9 & 277.0 & 766.4 \\
With PV plastic, mg & 79 & 557.5 & 54.3 & 5.5 & 189.9 & 458.4 & 769.3 & $2,593.7$ \\
Without PV plastic, pieces & 57 & 14.2 & 2.06 & 1.0 & 4.0 & 9.0 & 18.5 & 88.0 \\
With PV plastic, pieces & 79 & 26.9 & 2.39 & 1.0 & 12.0 & 24.0 & 37.3 & 104.0 \\
\hline Non-Juveniles & & & & & & & & \\
\hline Without PV plastic, mg & 21 & 134.1 & 34.0 & 4.2 & 23.2 & 62.1 & 185.7 & 532.0 \\
With PV plastic, mg & 10 & 219.1 & 79.7 & 8.0 & 29.4 & 93.3 & 486.3 & 677.0 \\
Without PV plastic, pieces & 21 & 8.05 & 1.34 & 1.00 & 3.00 & 7.0 & 13.5 & 22.0 \\
With PV plastic, pieces & 10 & 16.1 & 5.75 & 3.0 & 3.0 & 6.0 & 36.25 & 48.0 \\
\hline
\end{tabular}

In addition to an age difference, there was also a difference in gastric distribution related to time periods. Analyzing the two periods with large sample sizes of juveniles, 2010-2011 ( $n=69)$ and 20142015 ( $n=51$ ), significantly more juveniles contained stomach plastic in $2010-2011(94 \%, 65 / 69)$ than in 2014-2015 (78\%, 40/51), (Chi-square $=6.669, p=0.0098)$, but there was more plastic during the 2014-2015 time period (Table 6) (mass $W_{65,51}=3802, p=0.048$; number of pieces $W_{65,51}=3700$, 
$\mathrm{p}=0.012$ ). The distribution of plastic in stomachs differed significantly for the two time periods with $10 \%$ of pieces and $16 \%$ of mass of plastic in proventriculi in $2010-2011$ compared to $33 \%$ of pieces and $40 \%$ of mass in 2014-2015 (mass $W_{65,46}=824.5, p<0.0002$; number of pieces $W_{65,46}=873.5$, $p<0.0001)$. Overall, this indicates that fewer fulmars retained plastic in 2014-2015, but there was a shift with significantly more plastic in proventriculi in 2014-2015.

Table 6 Comparison of juvenile plastic loads for two time periods with the largest samples of juveniles. $\mathrm{N}=$ the number of stomachs in the sample, $\mathrm{Q} 1$ and $\mathrm{Q} 3$ are quartiles about the median, and "Total" is the combined plastic load for the proventriculus and ventriculus.

\begin{tabular}{llllllllll}
\hline Variable & Time Interval & N & Mean & SE & Min & Q1 & Median & Q3 & Max \\
\hline Total mg & $2010-2011$ & 69 & 426.3 & 69.4 & 0 & 90.0 & 209.0 & 545.5 & $2,608.0$ \\
& $2014-2015$ & 51 & $1,897.1$ & 657 & 0 & 57 & 557 & 1505 & $26,252.0$ \\
& & & & & & & & & \\
Total pieces & $2010-2011$ & 69 & 18.5 & 2.50 & 0 & 4 & 11 & 25.5 & 118 \\
& $2014-2015$ & 51 & 50.4 & 11.0 & 0 & 4 & 31 & 58.0 & 466 \\
\hline
\end{tabular}

We found plastic in $24.1 \%$ (13/54) of fulmar intestines from the 2014-2015 sample with a median in affected birds of two (number of pieces-number of samples 1-5, 2-4, 3-2, 8-1). The bird with eight pieces (specimen \#2017-108) retained a moderate load of $1,744.6 \mathrm{mg}$ in a pooled stomach sample. Intestinal pieces had a median greatest dimension of $3.0 \mathrm{~mm}$ (range 1.3-5.9 mm) and weight of 29 $\mathrm{mg}$ (range 0.9-117 $\mathrm{mg}$ ) and included hard plastic, assumed to be polypropylene or polyethylene, extruded foam, and one thread.

\section{Discussion}

\subsection{Fulmars from the NE Pacific Coast}

On the NE Pacific coast, juvenile fulmars represented $83 \%$ of the beached samples and averaged five times more plastic by mass than older birds. Juveniles retained more plastic in the expandable proventriculus than ventriculus whereas plastic in older birds was concentrated in the ventriculus. In a comparison of two time periods we also found a difference in the relative distribution of plastic between stomach sections in juveniles. Since proventricular plastic is held for a short term relative to ventricular plastic, variation in proventricular loads induces unknown variability when comparing samples, especially to Atlantic studies that are primarily ventricular plastic. Stomach-based plastic sampling would benefit for further study to elucidate causes for differences in age, gastric distribution, availability of oceanic plastic, and interaction among these factors.

\subsection{Demographics of Fulmar Plastic Samples}

Immature fulmars and other procellariids retain more plastic than adults $[1,10,13,17,20,24$, $26,37,38]$. Therefore, a predominance of immatures or adults could bias sample averages higher or lower, respectively, so the inclusion of demographic profiles are recommended for spatiotemporal comparisons $[1,10]$. The significance of demography was highlighted by a recent 
analysis of a 17-year data series from the North Sea that indicated the percent of fulmar adults in yearly samples added significantly to a model of a decreasing temporal trend in a proxy for plastic mass in stomachs $[6,9,10]$. This appears to indicate plastic loads were related to the percent of adults in pooled samples, confirming general conclusions [10].

Several other shorter-term studies are often used to assess the EcoQ\% performance in the North Atlantic or Arctic away from the North Sea (see Figure 3 in [10]). In these studies, age was categorized as adult versus immature but sample sizes were too small for comparisons [36, 39]. Adult/immature numbers were 49/9 from Iceland [39], 5/35 from Svalbard [36], and 61/9 for the Labrador Sea [7]. Bravo Rebolledo [21] from the Faroe Islands had adequate samples of 151 adults and 96 immatures (or non-adults) but did not statistically test the differences of $0.11 \mathrm{~g}$ in adults and $0.18 \mathrm{~g}$ in immatures. Bond et al. [40] examined 176 fulmars from Nova Scotia beaches from 20002012 and found no age difference but aging criteria or data were not provided. These studies have been used to assess EcoQ\% performance by pooling mass for age groups, but inclusion as age as related to plastic loads, as done of recently by van Franeker et al. [10] is recommended (see [1, 14, $15,41])$.

Few studies provided demographic profiles using three ages including juvenile, immature, and adult. Included is our study from the NE Pacific coast that had 83,5 , and $12 \%$, respectively, for three age classes (Table 3). Percentages for the same age classes in North Sea studies through the year 2000 were 24, 24, and 52 (Table 6 in [1]). As an example of bias from differing demographic profiles, an unweighted arithmetic mean was $0.83 \mathrm{~g}$ for the NE Pacific coast sample compared to $0.55 \mathrm{~g}$ (Figure 3a in [1]) for the North Sea from 1982 to 2000. If only adults were used, the trend is reversed with $0.22 \mathrm{~g}$ for the NE Pacific coast and $0.44 \mathrm{~g}$ for the North Sea through 2000 (Table 6 in [1]). North Sea levels have declined recently [10], but plastic associated with adults was not specified so updated comparisons weren't possible. As an assessment of the amount of plastic in Northern Fulmars as a species or population, the pooled estimate in the NE Pacific sample overestimates loads because of the predominance of juveniles in our samples while the majority of the population or species are adults [30, 42]. However, as long as demographic profiles are incorporated into assessments, sampling using stomachs provides some measure of environmental pollution for a region (see reviews $[4-6,9,10]$ ).

Several reasons have been advanced to account for larger plastic loads in immature birds as compared to adults. One suggestion, specific to recently fledged juveniles, was that larger loads represent plastic from parental transfer $[17,20]$. This seems negated by our findings that about half the juveniles had no more plastic than older birds and $14 \%$ contained no plastic. A related suggestion was that adults would contain smaller plastic loads due to "off-loading" while feeding young via regurgitation $[11,26]$. Off-loading would likely reduce passage and accumulation of plastic in the ventriculus in some adults, but only those that successfully fed young. This would leave $50 \%$ or more [30] of the adults with relatively large plastic loads due to lack of feeding young because of nesting failure. In any case, off-loading or regurgitation would not be confined to the chick feeding periods but probably occurs as needed to clear plastic and other indigestible matter from proventriculi [13]. This leaves a default assumption that adults are more selective foragers than immatures [17, 20] or the two groups forage in regions that differ in plastic availability [37]. 


\subsection{Gastric Distribution of Plastic in Fulmars}

Our NE Pacific fulmar sample appears to be unique in having a large proportion of plastic in proventriculi, especially in some juveniles. One other study examined the distribution of plastic in stomach sections separately using categories of adult versus non-adult from the Faroe Islands (North Atlantic) fulmars [21]. The percent of birds with proventricular plastic did not differ by age (adults $33 \%$ or 50 of 151, non-adult $42 \%$ or 40 of 96) (Table 4 in [21]) (Chi-square $=1.85, p=0.173$ ). For a direct comparison, we categorized our data in the same way (adult versus non-adult) and significantly fewer adults ( $27 \%$ or 6 of 22 ) contained proventricular plastic than non-adults ( $54 \%$ or 84 of 155) (Chi-square $=9.98, p=0.015$ ). A reason for a greater incidence of proventricular plastic in NE Pacific fulmars could be that pieces were larger averaging $8.3 \mathrm{~mm}$ greatest dimension and 65.6 $\mathrm{mg}$ [13] as compared to $6.95 \mathrm{~mm}$ and $20 \mathrm{mg}$ for the Faroe Island samples (Table 4, Figure 5 in [21]). Ventricular sizes were more similar averaging $5.5 \mathrm{~mm}$ and $25.3 \mathrm{mg}$ [13] and $5.65 \mathrm{~mm}$ and $11.8 \mathrm{mg}$ (Table 4, Figure 6 in [21]), which is an estimate of the approximate size that can squeeze through the proventricular-ventricular constriction [13, 14, 21]. Smaller pieces in Bravo Rebolledo [21] could pass into the ventriculus, while the larger pieces in NE Pacific fulmars may be too large to pass into the ventriculus thus accounting for large proventricular loads in NE Pacific fulmars..

As noted in the Introduction, a concentration of plastic in ventriculi indicates that proventriculi were cleared of plastic either through regurgitation or passage into the ventriculi. Although this is an obvious point, implications differ depending if plastic loads are used as bioindicators or are used to assess biological significance. For bioindicator use, capacity estimates based on maxima are with $2.6 \mathrm{~g}$ for ventriculi and almost 10 times more at $24.4 \mathrm{~g}$ for proventriculi (Table 3 ). In the context of these maxima, plastic loads are far below what a fulmar could hold (Table 3 ), especially given the amount of environmental plastic available $[12,43,44]$. Presumably, as the ventricular maximum nears or if pieces are too large to pass into the ventriculus, plastic accumulates in the proventriculus or is regurgitated. The difference in the accumulation of plastic in proventriculi in comparing the 2011-2012 to 2014-2015 time periods is unexplained but could reflect latency to regurgitate, size or age of particles, distance from pollution sources, foraging differences, or a combination of these ([45], also see Section 4.4, below). Proventricular plastic probably is recently acquired and retained for a short time relative to ventricular plastic [7, 13, 25]. Unlike our NE Pacific samples, the Atlantic samples are mostly ventricular $[1,2,7,10,21]$ so direct comparisons of pooled samples between the two oceans seems ill-advised.

\subsection{Changes in Oceanic Plastic and Gastrointestinal Dynamics}

Changes include a shift in the composition of stomach plastic from uniform pellets to irregularly shaped user particles in the 1990 s to 2000 s $[3,47,48]$. This shift was also evident in oceanic plankton tow-net sampling [44, 49-51]. In addition, as the mass of oceanic plastic ages, the number of pieces increases due to fragmentation with a corresponding reduction in size and increased accumulation of pieces less than $10 \mathrm{~mm}$ greatest dimension. Size varies with proximity to pollution sources, currents, exposure, temperature, and mixing [12, 44, 49, 51]. Unlike the pellet-to-user plastic shift, this change has not been considered in fulmar or other seabird studies. Most studies appear to assume that plastic size is constant and that stomach samples reflect changes in the availability of the amount of oceanic plastic (e.g., $[6,10])$. 
The significance of the shift in the size of user particles to stomach-based sampling is speculative. If fulmars selected particles in a certain size range, then stomach loads would sample changes in availability within the acceptable size range. If they ingest what was available, and particle size was reduced in time or regionally, then smaller particles might pass directly through the proventriculus into the ventriculus. If small enough, particles could pass immediately into the intestine and out of the birds. In planktonic sampling, the predominant particle size in the NW Atlantic is $<10 \mathrm{~mm}$ greatest dimension having changed from $10 \mathrm{~mm}$ to $5 \mathrm{~mm}$ through the 2000s [49] and continues to fragment [44]. Further north, in the Arctic Sea, the predominant size was 1-2 mm greatest dimension [51]. Based on the size of intestinal plastic $([13,21]$; this study) these small particles could pass through the gastrointestinal tracts. Since retained plastic is used for biomonitoring, the possibility that particle size is not constant in time or space should be considered along with demographics and gastric distribution of plastic. Extending this to differences in Pacific versus Atlantic, Atlantic plastic may be smaller pieces that are easily regurgitated or passed through into the ventriculus and intestine. This might account for the concentration of plastic in the ventriculus in Atlantic samples in contrast to samples from the NE Pacific coast.

\subsection{Conclusions}

Upon reconsideration, the NE Pacific Ocean plastic samples from fulmars do not appear to be directly comparable to those from Atlantic Ocean studies. This is due to a preponderance of juveniles along the NE Pacific coast that retained relatively large plastic loads, particularly in proventriculi. Our review demonstrates that the amount of plastic retained in samples can vary because of the expansive capacity of the proventriculus versus the limited capacity of the ventriculus. Unlike ventricular plastic, retention of proventricular plastic is relatively short-term before regurgitation or passage into the ventriculus. The NE Pacific coast samples may differ from the Atlantic in that proventricular pieces were larger than those in the Atlantic suggesting that environmental plastic pieces are larger in the NE Pacific, Pacific fulmars are selecting larger pieces, or both. Because of the preponderance of juveniles from the NE Pacific, loads for the population, region, and species are biased upward as are the use of pooled values in comparison to other regions. Based on a review of oceanic plastic, plastic input is either stable or increasing, while weathering of existing plastic is fragmenting and increasing the relative proportion of smaller pieces. This along with demographics and gastrointestinal dynamics could influence the utility of stomach plastic as bioindicators and change biological impacts.

\section{Acknowledgments}

This research did not receive any specific grants from funding agencies in the public, commercial, or not-for-profit sectors. We thank Jan A. van Franeker and two anonymous reviewers for comments, personnel of the Wildlife Center of the North Coast, Astoria, Oregon for assistance in procuring specimens, and University of Puget Sound students and staff including Lydia Kleine, Alicia Terepocki, Hannah Floren, Peter Wimberger, and Peter Hodum for specimen preparation and laboratory space. 


\section{Author Contributions}

Shugart conceived, preformed the analysis, and wrote the paper; Nania collected and summarized data and edited drafts.

\section{Competing Interests}

The authors have declared that no competing interests exist.

\section{References}

1. Van Franeker JA, Meijboom A. Litter NSV; marine litter monitoring by northern fulmars (a pilot study). Wageningen: Alterra; 2002; 401.

2. Van Franeker JA, Blaize C, Danielsen J, Fairclough K, Gollan J, Guse N, et al. Monitoring plastic ingestion by the northern fulmar Fulmarus glacialis in the North Sea. Environ Pollut. 2011; 159: 2609-2615.

3. Van Franeker JA, Law KL. Seabirds, gyres and global trends in plastic pollution. Environ Pollut. 2015; 203: 89-96.

4. OSPAR. Guidelines for monitoring and assessment of plastic particles in stomachs of fulmars in the North Sea area. OSPAR Commission Agreement 2015-03 (Source: ElHA 15/5/12 Add.1)[Internet]. Texel: OSPAR; $2015 . \quad$ Available from: http://www.ospar.org/convention/agreements?q=fulmar\&t=32281\&a=\&s.

5. Plastic particles in fulmar stomachs in the North Sea [Internet]. Texel: OSPAR Assessment Portal; 2017. Available from: https://oap.ospar.org/en/ospar-assessments/intermediate-assessment2017/pressures-human-activities/marine-litter/plastic-particles-fulmar-stomachs-north-sea/.

6. Plastic particles in fulmar stomachs in the North Sea [Internet]. Texel: OSPAR Assessment Portal; 2019. Available from: https://oap.ospar.org/en/ospar-assessments/committeeassessments/eiha-thematic-assessments/marine-litter/plastic-particles-in-fulmar-stomachsnorth-sea/.

7. Avery-Gomm S, Provencher JF, Liboiron M, Poon FE, Smith PA. Plastic pollution in the Labrador Sea: An assessment using the seabird northern fulmar Fulmarus glacialis as a biological monitoring species. Mar Pollut Bull. 2018; 127: 817-822.

8. Bonanno G, Orlando-Bonaca M. Ten inconvenient questions about plastics in the sea. Environ Sci Policy. 2018; 85: 146-154.

9. Van Franeker JA, Kühn S. Fulmar Litter EcoQO monitoring in the Netherlands-update 2019. Netherlands: Wageningen Marine Research; 2020; C074/20.

10. Van Franeker JA, Kühn S, Anker-Nilssen T, Edwards EW, Gallien F, Guse N, et al. New tools to evaluate plastic ingestion by northern fulmars applied to North Sea monitoring data 2002-2018. Mar Pollut Bull. 2021; 166: 112246.

11. Ryan PG. How quickly do albatrosses and petrels digest plastic particles? Environ Pollut. 2015; 207: 438-440.

12. Wilcox C, Van Sebille E, Hardesty BD. Threat of plastic pollution to seabirds is global, pervasive, and increasing. Proc Natl Acad Sci. 2015; 112: 11899-11904. 
13. Terepocki AK, Brush AT, Kleine LU, Shugart GW, Hodum P. Size and dynamics of microplastic in gastrointestinal tracts of Northern Fulmars (Fulmarus glacialis) and Sooty Shearwaters (Ardenna grisea). Mar Pollut Bull. 2017; 116: 143-150.

14. Nania TG, Shugart GW. Are plastic particles reduced in size in seabirds' stomachs? Mar Pollut Bull. 2021; 172: 112843.

15. Provencher JF, Bond AL, Avery-Gomm S, Borrelle SB, Rebolledo EL, Hammer S, et al. Quantifying ingested debris in marine megafauna: A review and recommendations for standardization. Anal Methods. 2017; 9: 1454-1469.

16. Connors PG, Smith KG. Oceanic plastic particle pollution: Suspected effect on fat deposition in red phalaropes. Mar Pollut Bull. 1982; 13: 18-20.

17. Day RH, Wehle DH, Coleman FC. Ingestion of plastic pollutants by marine birds. Proceedings of the Workshop on the Fate and Impact of Marine Debris; 1984 November 27-29; Honolulu, Hawaii. U.S.: NOAA.

18. Furness RW. Plastic particle pollution: Accumulation by Procellariiform seabirds at Scottish colonies. Mar Pollut Bull. 1985; 16: 103-106.

19. Van Franeker JA. Plastic ingestion in the North Atlantic fulmar. Mar Pollut Bull. 1985; 16: $367-$ 369.

20. Ryan PG. The effects of ingested plastic and other marine debris on seabirds. Proceedings of the Second International Conference on Marine Debris; 1989 April 2-7; Honolulu, Hawaii, US. Washington, D.C.: National Oceanic and Atmospheric Administration. NMFS.NOAA-TM-NMFSSWFSC-154.199.

21. Bravo Rebolledo E. Threshold levels and size dependent passage of plastic litter in stomachs of Fulmars. Wageningen, Gelderland, Netherlands: Wageningen University; 2011.

22. Furness RW. Ingestion of plastic particles by seabirds at Gough Island, South Atlantic Ocean. Environ Pollut. 1985; 38: 261-272.

23. Moser ML, Lee DS. A fourteen-year survey of plastic ingestion by western North Atlantic seabirds. Colon Waterbirds. 1992; 15: 83-94.

24. Robards MD, Gould PJ, Piatt JF. The highest global concentrations and increased abundance of oceanic plastic debris in the North Pacific: Evidence from seabirds. In: Marine debris. New York: Springer; 1997. p. 71-80.

25. Ask A, Cusa M, Danielsen J, Gabrielsen GW, Strand J. Plastic characterization in northern fulmars (Fulmarus glacialis). Nordic Council of Ministers; 2020.

26. Ryan PG. Intraspecific variation in plastic ingestion by seabirds and the flux of plastic through seabird populations. Condor. 1988; 90: 446-452.

27. Spear LB, Ainley DG, Ribic CA. Incidence of plastic in seabirds from the tropical pacific, 19841991: Relation with distribution of species, sex, age, season, year and body weight. Mar Environ Res. 1995; 40: 123-146.

28. Van Franeker J. Save the North Sea Fulmar-Litter-EcoQO manual part 1: Collection and dissection procedures. Wageningen: Alterra; 2004; 672.

29. Glick B. Bursa of fabricius. In: Avian biology. Cambridge, Massachusetts: Academic Press; 1983.

30. Mallory ML, Hatch SA, Nettleship DN. Northern fulmar. Fulmarus glacialis. Ithaca, NY, USA: Cornell Lab of Ornithology; 2020. Doi: 10.2173/bow.norful.01.

31. Dawson A. Control of the annual cycle in birds: Endocrine constraints and plasticity in response to ecological variability. Philos Trans R Soc B Biol Sci. 2007; 363: 1621-1633. 
32. Broughton JM. Size of the bursa of Fabricius in relation to gonad size and age in Laysan and Black-footed Albatrosses. Condor. 1994; 96: 203-207.

33. Minitab 17.3.1 Statistical Software. State College, PA: Minitab, Inc; 2016. Available from: https://www.minitab.com.

34. Zar JH. Biostatistical analysis. In: Upper saddle river. Hoboken, New Jersey: Prentice-Hall, Inc.; 1999.

35. Mallory ML, Roberston GJ, Moenting A. Marine plastic debris in northern fulmars from Davis Strait, Nunavut, Canada. Mar Pollut Bull. 2006; 52: 813-815.

36. Trevail AM, Gabrielsen GW, Kühn S, Van Franeker JA. Elevated levels of ingested plastic in a high Arctic seabird, the northern fulmar (Fulmarus glacialis). Polar Biol. 2015; 38: 975-981.

37. Ogi H. Ingestion of plastic particles by Sooty and Short-tailed Shearwaters in the North Pacific. Proceedings of the Second International Conference on Marine Debris; 1989 April 2-7; Honolulu, Hawaii.

38. Carey MJ. Intergenerational transfer of plastic debris by Short-tailed Shearwaters (Ardenna tenuirostris). Emu. 2011; 111: 229-234.

39. Kühn S, van Franeker JA. Plastic ingestion by the northern fulmar (Fulmarus glacialis) in Iceland. Mar Pollut Bull. 2012; 64: 1252-1254.

40. Bond AL, Provencher JF, Daoust PY, Lucas ZN. Plastic ingestion by fulmars and shearwaters at Sable Island, Nova Scotia, Canada. Mar Pollut Bull. 2014; 87: 68-75.

41. Provencher JF, Bond AL, Hedd A, Montevecchi WA, Muzaffar SB, Courchesne SJ, et al. Prevalence of marine debris in marine birds from the North Atlantic. Mar Pollut Bull. 2014; 84: 411-417.

42. Hatch SA. Adult survival and productivity of Northern Fulmars in Alaska. Condor. 1987; 89: 685696.

43. Eriksen M, Lebreton LC, Carson HS, Thiel M, Moore CJ, Borerro JC, et al. Plastic pollution in the world's oceans: More than 5 trillion plastic pieces weighing over 250,000 tons afloat at sea. PloS one. 2014; 9: e111913.

44. Wilcox C, Hardesty BD, Law KL. Abundance of floating plastic particles is increasing in the Western North Atlantic Ocean. Environ Sci Technol. 2020; 54: 790-796.

45. Roman L, Hardesty BD, Hindell MA, Wilcox C. Disentangling the influence of taxa, behaviour and debris ingestion on seabird mortality. Environ Res Lett. 2020; 15: 124071.

46. Ryan PG. Ingestion of plastics by marine organisms. In: Hazardous chemicals associated with plastics in the marine environment. New York: Springer; 2016. p. 235-266.

47. Vlietstra LS, Parga JA. Long-term changes in the type, but not amount, of ingested plastic particles in short-tailed shearwaters in the southeastern Bering Sea. Mar Pollut Bull. 2002; 44: 945-955.

48. Ryan PG. Seabirds indicate changes in the composition of plastic litter in the Atlantic and southwestern Indian Oceans. Mar Pollut Bull. 2008; 56: 1406-1409.

49. Morét-Ferguson S, Law KL, Proskurowski G, Murphy EK, Peacock EE, Reddy CM. The size, mass, and composition of plastic debris in the western North Atlantic Ocean. Mar Pollut Bull. 2010; 60: 1873-1878.

50. Law KL, Morét-Ferguson S, Maximenko NA, Proskurowski G, Peacock EE, Hafner J, et al. Plastic accumulation in the North Atlantic subtropical gyre. Science. 2010; 329: 1185-1188. 
51. Cózar A, Martí E, Duarte CM, García-de-Lomas J, Van Sebille E, Ballatore TJ, et al. The Arctic Ocean as a dead end for floating plastics in the North Atlantic branch of the Thermohaline Circulation. Sci Adv. 2017; 3: e1600582.

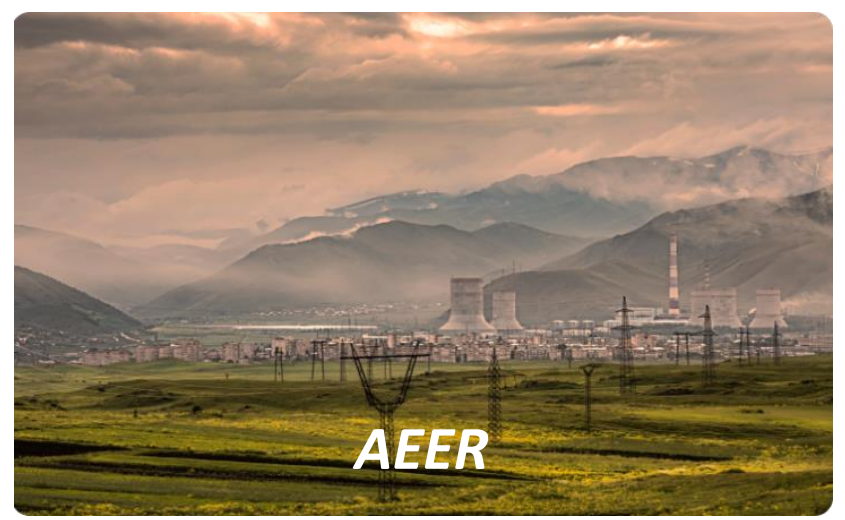

Enjoy AEER by:

1. Submitting a manuscript

2. Joining in volunteer reviewer bank

3. Joining Editorial Board

4. Guest editing a special issue

For more details, please visit:

http://www.lidsen.com/journals/aeer 